\title{
How basin stability complements the linear-stability paradigm
}

\author{
Peter J. Menck ${ }^{1,2 \star}$, Jobst Heitzig ${ }^{1}$, Norbert Marwan ${ }^{1}$ and Jürgen Kurths ${ }^{1,2,3}$
}

The human brain ${ }^{1,2}$, power grids ${ }^{3}$, arrays of coupled lasers ${ }^{4}$ and the Amazon rainforest ${ }^{5,6}$ are all characterized by multistability ${ }^{7}$. The likelihood that these systems will remain in the most desirable of their many stable states depends on their stability against significant perturbations, particularly in a state space populated by undesirable states. Here we claim that the traditional linearization-based approach to stability is too local to adequately assess how stable a state is. Instead, we quantify it in terms of basin stability, a new measure related to the volume of the basin of attraction. Basin stability is non-local, nonlinear and easily applicable, even to high-dimensional systems. It provides a long-sought-after explanation for the surprisingly regular topologies ${ }^{8-10}$ of neural networks and power grids, which have eluded theoretical description based solely on linear stability ${ }^{11-13}$. We anticipate that basin stability will provide a powerful tool for complex systems studies, including the assessment of multistable climatic tipping elements ${ }^{14}$.

Complex systems science relies heavily on linear stability analysis, in which state of a dynamic system (more correctly, its dynamic regime) is assessed basically by inspecting the dominant curvature of the potential energy function in the state's surroundings (as expressed by Lyapunov exponents). The absolute value of the curvature measures the speed of convergence or divergence after a small perturbation, and its sign qualifies the state as stable or unstable. Such linearization-based considerations are inherently local; therefore, they are not sufficient to quantify how stable a state is against non-small perturbations. Quantification of stability in this sense requires a global concept: the basin of attraction $\mathcal{B}$ of a state is the set of initial points in state space from which the system converges to this state. Complete knowledge of the basin would allow us to fully assess the state's stability: one could classify perturbations into the permissible and the impermissible. See Fig. 1.

However, basins are intricate entities ${ }^{15}$ and especially hard to explore in high dimensions. Here we therefore focus on a single but fundamental property: the basin's volume. The authors of ref. 16 interpret the volume of a state's basin of attraction as a measure of the likelihood of arrival at this state, that is, as a measure of the state's relevance. Almost equivalently, we understand the volume of the basin as an expression of the likelihood of return to the state after any random-possibly non-small-perturbation. This yields a second interpretation: the basin's volume quantifies how stable a state is. To the best of our knowledge, this interpretation has not yet been employed in complex systems science. We refer to the quantification of stability based on the basin's volume as basin stability $S_{\mathcal{B}}$.

For climatic tipping elements ${ }^{14}$ it would be particularly useful to know how stable the desirable (that is, present) state is against

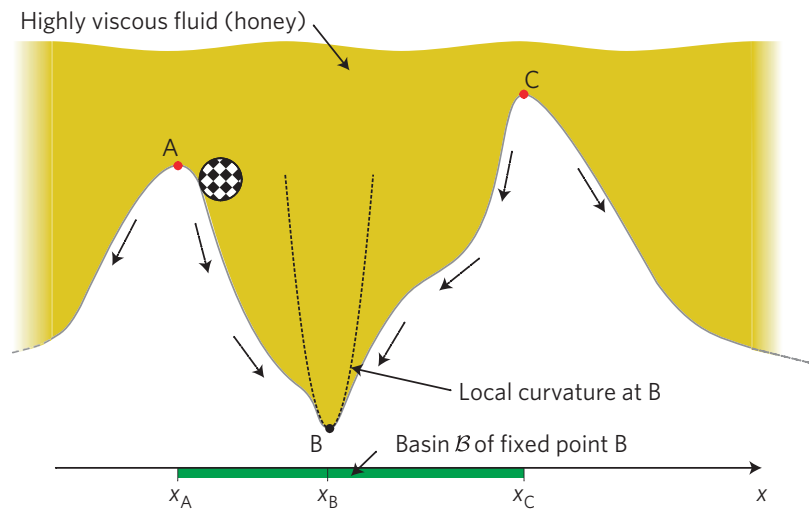

Figure 1 | Thought experiment: marble on a marble track. The track is immersed in a highly viscous fluid to make the system's state space one-dimensional. Dashed arrows indicate where the marble would roll from each position. A, B and C label fixed points. Only B is stable. The green bar indicates $B$ 's basin of attraction $\mathcal{B}$. If the marble is perturbed from $B$ to $a$ state within the basin, it will return to $B$. Such perturbations are permissible. Perturbations to states outside the basin are impermissible. The dashed parabola shows the local curvature around B, fitting the true marble track poorly in most of the basin.

perturbations. One such tipping element is the Amazon rainforest, which presumably possesses two stable states: the present fertile forest state and a barren savanna state ${ }^{5,6}$. A transition would emit huge amounts of carbon dioxide captured in the rich vegetation. Amazonian bistability arises from a positive feedback: deep-rooting trees take up water stored in the soil and transpire it to the atmosphere. Thereby, forest cover in an area increases overall precipitation and improves its own growing conditions. Consequently, a rather arid area (that is, an area with weak precipitation inflow) may still be supportive of forest growth if its forest cover exceeds a certain critical threshold; were forest cover pushed below this threshold, the area would lose all of its trees.

This is summarized in a conceptual model (see Supplementary Information),

$$
\frac{\mathrm{d} C}{\mathrm{~d} t}=F(C)=\left\{\begin{aligned}
r(1-C) C-x C & \text { if } C>C_{\text {crit }} \\
-x C & \text { if } C<C_{\text {crit }}
\end{aligned}\right.
$$

Here, $C$ is the relative forest cover that grows with the saturating rate $r$ if $C>C_{\text {crit }}$ and dies with rate $x$ (assuming $r>x>0$ ). $C_{\text {crit }}$ is the critical forest cover threshold. This model has two equilibria, the forest state $C_{\mathrm{F}}=1-x / r$ and the savanna state $C_{\mathrm{S}}=0$. The

${ }^{1}$ Research Domain on Transdisciplinary Concepts and Methods, Potsdam Institute for Climate Impact Research, PO Box 6012 03, 14412 Potsdam, Germany, ${ }^{2}$ Department of Physics, Humboldt University of Berlin, Newtonstraße 15, 12489 Berlin, Germany, ${ }^{3}$ Institute for Complex Systems and Mathematical Biology, University of Aberdeen, Aberdeen AB24 3UE, UK. *e-mail: menck@pik-potsdam.de. 


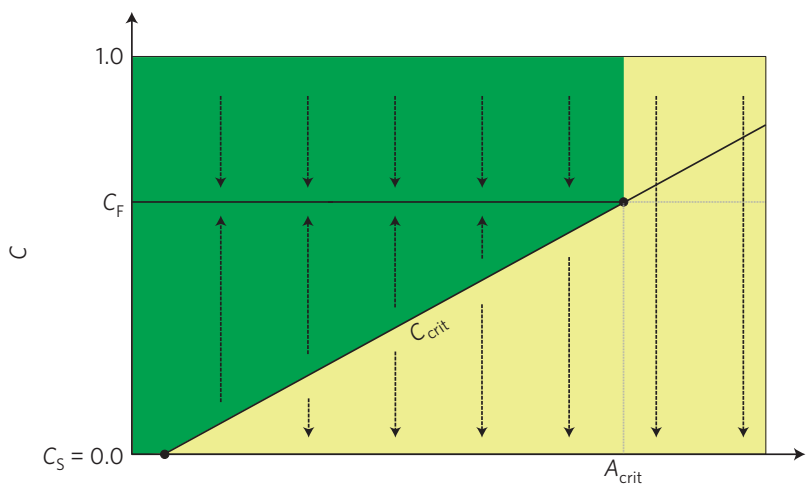

$A$

Figure $\mathbf{2}$ | State diagram of a bistable stylized forest-savanna model. Dashed arrows show where the system state, forest cover $C$, would move from a certain initial state at given aridity $A$. The green (light yellow) area is the basin of attraction of the forest (savanna) state $C_{F}\left(C_{S}\right)$. Solid circles indicate the emergence or disappearance of an equilibrium.

equilibrium $C_{\mathrm{F}}$ (respectively, $C_{\mathrm{S}}$ ) exists and is stable if $C_{\mathrm{F}}>C_{\text {crit }}$ (respectively, $C_{\text {crit }}>0$ ). Assuming that $C_{\text {crit }}$ increases linearly with aridity $A$, we obtain the state diagram shown in Fig. 2.

Global warming may drive up aridity, pushing it eventually beyond the bifurcation point $A_{\text {crit }}$ where the forest state vanishes. As aridity increases, the volume of the $C_{\mathrm{F}}$ state's basin of attraction shrinks, indicating that the forest state becomes less stable against perturbations. Indeed, owing to this reduced basin stability, a large perturbation such as strong deforestation might push the system to the savanna state long before aridity reaches $A_{\text {crit }}$. Crucially, none of this is reflected by linear stability, which judges the forest state $C_{\mathrm{F}}$ only by the stability coefficient $F^{\prime}\left(C_{\mathrm{F}}\right)=x-r$ that remains constant as aridity goes up.

This implies, first, that there is no critical slowing down. Indeed, linear early-warning signals ${ }^{17}$ can be absent in systems with strong nonlinearities such as this model. Second, because of their highly local perspective, linear stability and hence the small-perturbation convergence rate do not indicate how stable $C_{\mathrm{F}}$ is against non-small perturbations. They are unreliable proxies, in contradiction to previous observations ${ }^{17,18}$.

Note that both effects may also emerge in such simple mechanical systems as a damped driven pendulum (see Supplementary Information). Clearly, global stability concepts are needed.

Such global stability concepts could incorporate potential energy functions: deeper valleys would correspond to more stable states (Fig. 1). However, energy functions may not be available for many relevant (dissipative) systems. Furthermore, the estimation of energy levels on the basin boundary is numerically costly in high dimensions. An alternative approach was suggested, globally quantifying stability (or resilience, in the original nomenclature) in terms of the width of the basin of attraction in a particular direction ${ }^{19,20}$. This concept has inspired a host of studies on complex socioecological systems. Yet the measure of stability it implies "is not so easily quantified, even in models"18. One reason is that, in systems with many state variables, it is impossible to identify the single most relevant direction along which the width of the basin should be gauged. Our basin stability offers two important improvements: first, it follows a volume-based probabilistic approach that is compatible with the natural uncertainty about the strength and direction of perturbations; second, it provides a measure of stability that is clearly defined and easily quantified even in high-dimensional systems (see Methods).

Basin stability's applicability to high-dimensional systems allows us to tackle a puzzle that has long haunted complex networks science. Researchers in this field strive to understand how a network's topology serves its function and robustness ${ }^{21,22}$. Special effort has been put into multistable dynamic networks in which a synchronous state competes with alternative non-synchronous or partly synchronous states ${ }^{11-13,23}$. In a power grid, for instance, all components have to be operated at the same synchronous frequency to achieve steady power flows and to avoid damaging resonance effects $^{3}$. In the brain, both neural communication ${ }^{24}$ and memory processes ${ }^{25,26}$ rely vitally on the synchronous firing of neurons. This means that, although synchronization is also associated with pathological states such as Parkinsonian tremor ${ }^{27}$, the functional ability to support synchrony is as pivotal for the brain as it is for power grids. The problem with both kinds of network is that their real-world topologies look completely different from what the theory predicts.

The theory is as follows. If the synchronous state of a dynamic network is to be maintained, it must be stable against perturbations. A groundbreaking study ${ }^{11}$ based on linearization revealed that, for a network of identical oscillators, the stability of the synchronous state can directly be inferred from the Laplacian, a matrix that reflects the coupling topology (see Supplementary Information). Indeed, for many types of oscillator, the synchronous state is stable if the ratio of the Laplacian's maximum and minimum non-zero eigenvalues, $R=\lambda_{\max } / \lambda_{\min }$, is smaller than an oscillator-specific stability threshold, $\beta=\alpha_{2} / \alpha_{1}$, provided the coupling strength is chosen from the stability interval, $I_{\mathrm{s}}=\left(\alpha_{1} / \lambda_{\min }, \alpha_{2} / \lambda_{\max }\right)$. The ratio $R$ is known as the synchronizability of a network. Networks with smaller $R$ are considered more synchronizable ${ }^{12}$. To determine what particularly synchronizable networks look like, researchers ${ }^{13}$ employed the Watts-Strogatz graph generation model ${ }^{8}$ and found that, as the model is tuned from regular lattices (model parameter $p=0)$ to random graphs $(p=1)$, synchronizability shows a strong, monotonical improvement (Fig. 3a).

Consequently, according to linear-stability-based synchronizability, real-world networks whose function relies on synchronization should ideally look like random graphs. However, neural networks and power grids exhibit small-world topologies that, from the Watts-Strogatz model's perspective, are far more regular than random graphs ${ }^{8-10}$. Indeed, when building well-functioning synchronizable networks, nature and civilization seem to shun the predicted randomness. This discrepancy between theory and observation has left networks research with a long-standing puzzle.

Attempting to complement the theory, we applied basin stability to ensembles of Watts-Strogatz networks consisting of paradigmatic Rössler oscillators, in which the dynamics at node $i$ obey

$$
\begin{gathered}
\dot{x}_{i}=-y_{i}-z_{i}-K \sum_{j=1}^{N} L_{i j} x_{j} \\
\dot{y}_{i}=x_{i}+a y_{i} \\
\dot{z}_{i}=b+z_{i}\left(x_{i}-c\right)
\end{gathered}
$$

with coupling constant $K$, Laplacian matrix $L, a=b=0.2$, and $c=7.0$. Every such network has a synchronous state in which all nodes follow the same trajectory. A network's synchronous state is stable if its synchronizability $R<\alpha_{2} / \alpha_{1}=37.85$ and $K \in I_{\mathrm{s}}=\left(\alpha_{1} / \lambda_{\min }, \alpha_{2} / \lambda_{\max }\right)$, where $\alpha_{1}=0.1232$ and $\alpha_{2}=4.663$. However, the level of $R$ does not quantify how stable the synchronous state is against perturbations. To address this yet unasked question, for each network we estimated the synchronous state's basin stability $S_{\mathcal{B}}$ for several $K \in I_{\mathrm{S}}$ and computed the mean $\bar{S}_{\mathcal{B}}=\operatorname{mean}\left\langle S_{\mathcal{B}}(K)\right\rangle_{K \in I_{\mathrm{s}}}$ (see Methods). Finally, we averaged $\bar{S}_{\mathcal{B}}$ over the ensemble to obtain the expected basin stability $\left\langle\bar{S}_{\mathcal{B}}\right\rangle$. We found that, in sharp contrast to synchronizability, expected basin stability declines exponentially fast as networks become more random (Fig. 3a,b; for a qualitative explanation, see Supplementary Information). Therefore, the synchronous state is much more stable in networks that are more regular. 


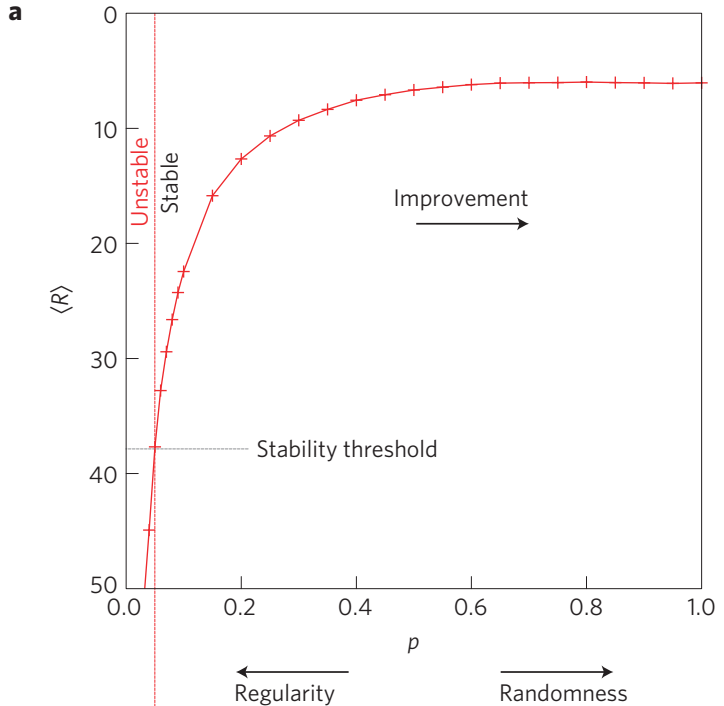

b

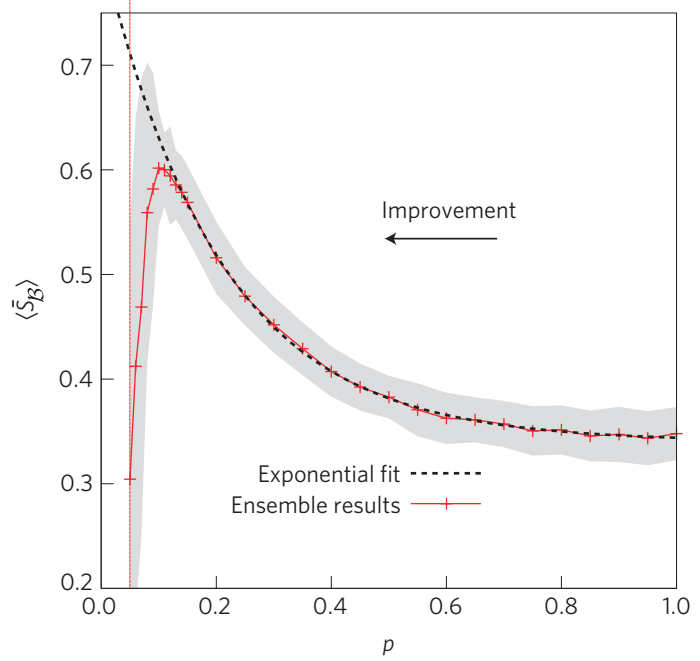

Figure 3 | Synchronizability and basin stability in Watts-Strogatz networks of chaotic oscillators. a, Expected synchronizability $\langle R\rangle$ versus the Watts-Strogatz model's parameter $p$. The scale of the $y$ axis was reversed to indicate improvement on increase in $p$. $\mathbf{b}$, Expected basin stability $\left\langle\bar{S}_{\mathcal{B}}\right\rangle$ versus $p$. The grey shading indicates \pm one standard deviation. The dashed line shows an exponential curve fitted to the ensemble results for $p \geq 0.15$. Solid lines are guides to the eye. The plots shown were obtained for $N=100$ oscillators of Rössler type, each having on average $k=8$ neighbours. Choices of larger $N$ and different $k$ produce results that are qualitatively the same. See Methods and Supplementary Information for details and a qualitative explanation of the main characteristics.

This adds a crucial piece to the puzzle and, we conjecture, makes its solution emerge (Fig. 4): in synchronizing networks, the functional need for the synchronous state to be as stable as possible promotes topological regularity. Thus, during network evolution, the optimization of synchronizability and the simultaneous optimization of basin stability have acted as two opposing forces. Their contest ended in a topological tradeoff: small-worldness.

Here, we have introduced basin stability, a new universal concept of stability. We see many important applications, notably cell regulatory networks, whose carcinogenic gene expression profiles have been related to cancer attractors in a high-dimensional multistable state space ${ }^{28}$. Like linear stability, basin stability is a property of a deterministic system and contains no information on the external perturbations that may affect it. Hence, it may

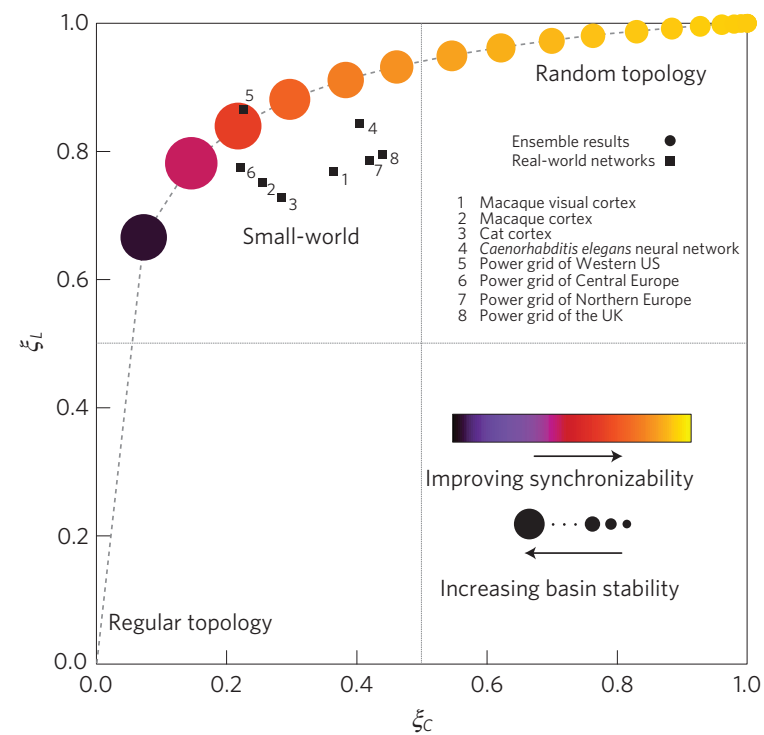

Figure 4 | Topological comparison of ensemble results with real-world networks. Circles represent the results for Watts-Strogatz networks with $N=100, k=10$ and rewiring probability $p \in\{0.05,0.1,0.15, \ldots, 1.0\}(p$ increasing from left to right). Circle area is proportional to the expected basin stability $\left\langle\bar{S}_{\mathcal{B}}\right\rangle$, and colour indicates the expected synchronizability $\langle R\rangle$. Squares represent real-world networks reported to exhibit a small-world topology (Supplementary Table S1). We chose $\xi_{L}$ and $\xi_{C}$ so that networks of different sizes can be compared with respect to average shortest path $L$ and clustering coefficient $C$, quantities that characterize small-worldness ${ }^{8}$. $\left(\xi_{L}, \xi_{C}\right)=(0,0)$ labels a regular network whereas $\left(\xi_{L}, \xi_{C}\right)=(1,1)$ labels a random network. Small-world networks reside in the top-left quadrant. See Methods and Supplementary Information.

often be promising to conceptually combine basin stability with a non-uniform distribution of perturbations. Basin stability could also be applied to stochastic systems by incorporating a suitable probabilistic notion of basins.

\section{Methods}

Estimation of basin stability in Watts-Strogatz networks of Rössler oscillators. A Watts-Strogatz network is constructed as follows ${ }^{8}$ : starting from a one-dimensional ring of $N$ nodes in which every node is connected to its $k$ nearest neighbours, each edge is rewired independently with probability $p$ by re-choosing one of its endpoints randomly. A Watts-Strogatz network generated with $p=0$ $(p=1)$ has a regular (random) topology. An intermediate value of $p$ yields a small-world topology.

In a given network, the dynamics at node $i(i=1, \ldots, N)$ are governed by the coupled Rössler equations (1)-(3). For $K \in I_{\mathrm{s}}:=\left(0.1232 / \lambda_{\min }, 4.663 / \lambda_{\max }\right)$, we want to estimate the volume of the synchronous state's basin of attraction $\mathcal{B}$. High dimensionality poses challenges. If $\mathcal{B}$ were a bounded convex set, its volume could be estimated in $\mathcal{O}\left(n^{4}\right)$ time steps with today's best algorithm ${ }^{29}$, where $n=3 \mathrm{~N}$ is the dimension of state space. We have $N \geq 100$, so this would be numerically very expensive. In any case, $\mathcal{B}$ is not convex in Rössler networks (see Supplementary Information). Thus, we retreat to something feasible: we estimate the volume of $\mathcal{B}$ in a relative sense, measuring basin stability as $S_{\mathcal{B} \cap \mathcal{Q}}=\operatorname{Vol}(\mathcal{B} \cap \mathcal{Q}) / \operatorname{Vol}(\mathcal{Q}) \in[0,1]$, where $\mathcal{Q}$ is a subset of state space that has finite volume.

More specifically, we integrate the system equations for $T$ initial conditions drawn uniformly at random from $\mathcal{Q}$. We count the number $M$ of initial conditions that arrive at the synchronous state (the other possible attractor being infinity) and estimate $S_{\mathcal{B} \cap \mathcal{Q}}$ as $M / T$. Observing that this is a repeated Bernoulli experiment, we infer that the estimate carries a standard error of

$$
e:=\frac{\sqrt{S_{\mathcal{B} \cap \mathcal{Q}}\left(1-S_{\mathcal{B} \cap \mathcal{Q})}\right.}}{\sqrt{T}}
$$

At $T=500$, for example, $e<0.023$ in absolute terms.

In relative terms, $e<S_{\mathcal{B} \cap \mathcal{Q}} / 10$ for $S_{\mathcal{B} \cap \mathcal{Q}}>1 / 6$. $\mathcal{Q}$ should be chosen such that values of $S_{\mathcal{B} \cap \mathcal{Q}}$ typically surpass this level. Our choice underlying the results presented above is $\mathcal{Q}=q^{N}$ with $q=[-15,15] \times[-15,15] \times[-5,35]$. Note that the Rössler attractor is included in $q$. We also studied other choices 
of $\mathcal{Q}-$ for instance $([-8,8] \times[-8,8] \times[-8,8])^{N}-$ yet observed no qualitative difference in the outcomes. Therefore, we suppress the subscript $\mathcal{Q}$ when stating $S_{\mathcal{B}}$ in Fig. 3.

For each network in the ensemble, we estimate basin stability $S_{\mathcal{B}}$ for ten different equally spaced values $K \in I_{\mathrm{s}}$ and average to obtain mean basin stability $\bar{S}_{\mathcal{B}}$.

The results are not qualitatively different for networks produced by a two-dimensional Watts-Strogatz model and another model that varies the link length distribution ${ }^{30}$. Details on this are provided in the Supplementary Information.

Topological comparison of small-world networks of different sizes. The axes of Fig. 4 were chosen so that different real-world networks can be compared, without the distorting effects of network size, with respect to average shortest path $L$ and clustering coefficient $C . L$ and $C$ have been widely used to characterize small-worldness ${ }^{8}$. We plot $\xi_{L}$ against $\xi_{C}$, where $\xi_{X}=1-\log \left(X / X_{\mathrm{R}}\right) / \log \left(X_{\mathrm{L}} / X_{\mathrm{R}}\right)$ with $X=L$ or $C$. $L_{\mathrm{R}}, C_{\mathrm{R}}\left(\right.$ or $\left.L_{\mathrm{L}}, C_{\mathrm{L}}\right)$ are the values of the respective quantities in random networks (or regular lattices) of the same size. $\xi_{X}$ counts how many orders of magnitude $X$ is away from $X_{\mathrm{L}}$ in relation to the count of orders of magnitude between $X_{\mathrm{L}}$ and $X_{\mathrm{R}}$. This way $\left(\xi_{L}, \xi_{C}\right)=(0,0)$ labels a regular network whereas $\left(\xi_{L}, \xi_{C}\right)=(1,1)$ labels a random network. Small-world networks have ${ }^{8} L \approx L_{\mathrm{R}}$ and $C \gg C_{\mathrm{R}}$ and therefore reside in the top-left quadrant. See Supplementary Information.

Received 24 July 2012; accepted 22 November 2012; published online 6 January 2013

\section{References}

1. Babloyantz, A. \& Destexhe, A. Low-dimensional chaos in an instance of epilepsy. Proc. Natl. Acad. Sci. USA 83, 3513-3517 (1986).

2. Lytton, W. W. Computer modelling of epilepsy. Nature Rev. Neurosci. 9, 626-637 (2008).

3. Machowski, J., Bialek, J. W. \& Bumby, J. R. Power System Dynamics: Stability and Control (Wiley, 2008).

4. Erzgräber, H. et al. Mutually delay-coupled semiconductor lasers: Mode bifurcation scenarios. Opt. Commun. 255, 286-296 (2005).

5. Da Silveira Lobo Sternberg, L. Savanna-forest hysteresis in the tropics. Glob. Ecol. Biogeogr. 10, 369-378 (2001).

6. Hirota, M., Holmgren, M., Van Nes, E. H. \& Scheffer, M. Global resilience of tropical forest and savanna to critical transitions. Science 334, 232-235 (2011).

7. May, R. M. Thresholds and breakpoints in ecosystems with a multiplicity of stable states. Nature 269, 471-477 (1977).

8. Watts, D. J. \& Strogatz, S. H. Collective dynamics of 'small-world' networks. Nature 393, 440-442 (1998)

9. Sporns, O. \& Zwi, J. The small world of the cerebral cortex. Neuroinformatics 2, 145-162 (2004).

10. Wang, Z., Scaglione, A. \& Thomas, R. J. Generating statistically correct random topologies for testing smart grid communication and control networks. IEEE Trans. Smart Grid 1, 28-39 (2010).

11. Pecora, L. M. \& Carroll, T. L. Master stability functions for synchronized coupled systems. Phys. Rev. Lett. 80, 2109-2112 (1998).

12. Barahona, M. \& Pecora, L. M. Synchronization in small-world systems. Phys. Rev. Lett. 89, 054101 (2002).

13. Hong, H., Kim, B. J., Choi, M. Y. \& Park, H. Factors that predict better synchronizability on complex networks. Phys. Rev. E 69, 067105 (2004).
14. Lenton, T. M. et al. Tipping elements in the earth's climate system. Proc. Natl. Acad. Sci. USA 105, 1786-1793 (2008).

15. Nusse, H. E. \& Yorke, J. A. Basins of attraction. Science 271, 1376-1380 (1996).

16. Wiley, D. A., Strogatz, S. H. \& Girvan, M. The size of the sync basin. Chaos 16, 015103 (2006).

17. Scheffer, M. et al. Early-warning signals for critical transitions. Nature 461, 53-59 (2009).

18. Van Nes, E. H. \& Scheffer, M. Slow recovery from perturbations as a generic indicator of a nearby catastrophic shift. Am. Nature 169, 738-747 (2007).

19. Holling, C. S. Resilience and stability of ecological systems. Annu. Rev. Ecol. Syst. 4, 1-23 (1973)

20. Scheffer, M. Critical Transitions in Nature and Society (Princeton Univ. Press, 2009).

21. Boccaletti, S., Latora, V., Moreno, Y., Chavez, M. \& Hwang, D-U. Complex networks: Structure and dynamics. Phys. Rep. 424, 175-308 (2006).

22. Buldyrev, S. V., Parshani, R., Paul, G., Stanley, H. E. \& Havlin, S. Catastrophic cascade of failures in interdependent networks. Nature 464, 1025-1028 (2010).

23. Arenas, A., Díaz-Guilera, A., Kurths, J., Moreno, Y. \& Zhou, C. Synchronization in complex networks. Phys. Rep. 469, 93-153 (2008).

24. Fries, P. A mechanism for cognitive dynamics: neuronal communication through neuronal coherence. Trends Cogn. Sci. 9, 474-480 (2005).

25. Fell, J. et al. Human memory formation is accompanied by rhinal-hippocampal coupling and decoupling. Nature Neurosci. 4, 1259-1264 (2001).

26. Fell, J. \& Axmacher, N. The role of phase synchronization in memory processes. Nature Rev. Neurosi. 12, 105-118 (2011).

27. Hammond, C., Bergman, H. \& Brown, P. Pathological synchronization in Parkinson's disease: Networks, models and treatments. Trends Neurosci. 30, 357-364 (2007).

28. Huang, S. \& Ingber, D.E. A non-genetic basis for cancer progression and metastasis: Self-organizing attractors in cell regulatory networks. Breast Disease 26, 27-54 (2007)

29. Lovász, L. \& Vempala, S. Simulated annealing in convex bodies and an $\mathcal{O}^{*}\left(n^{4}\right)$ volume algorithm. J. Comput. Syst. Sci. 72, 392-417 (2006).

30. Li, G. et al. Towards design principles for optimal transport networks. Phys. Rev. Lett. 104, 018701 (2010).

\section{Acknowledgements}

The authors acknowledge financial support from IRTG 1740 (Deutsche Forschungsgemeinschaft), the SUMO-project (European Union), the ECONS-project (Leibniz Association) and Konrad-Adenauer-Stiftung. They thank N. Fujiwara and A. Rammig for inspiring discussions.

\section{Author contributions}

P.J.M. conceived the study, performed the numerics, and prepared the manuscript. All authors discussed the results, drew conclusions and edited the manuscript. J.K. supervised the study.

\section{Additional information}

Supplementary information is available in the online version of the paper. Reprints and permissions information is available online at www.nature.com/reprints. Correspondence and requests for materials should be addressed to P.J.M.

\section{Competing financial interests}

The authors declare no competing financial interests. 